\title{
Verbal violence at work place: A reality from Pakistani context
}

\author{
Laila Akber Cassum \\ School of Nursing and Midwifery, Aga Khan University, Karachi, Pakistan.
}

Correspondence: Laila Akber Cassum. Address: School of Nursing and Midwifery, Aga Khan University, Karachi, Pakistan. Email: laila.akber.mn12@student.aku.edu

Received: March 3, 2014

DOI : $10.5430 /$ jnep.v4n8p20

Accepted: March 25, 2014

URL: http://dx.doi.org/10.5430/jnep.v4n8p20

\section{Abstract}

Work place violence is the most distressing subject globally, and has been a concern for many decades affecting home and work environment. This cancer has even invaded health care sector worldwide. Nursing professionals whether they are at hospital or at academics institutions are subjected to violence. Although, it has been a burning issue, among nursing health care workers it has often gone unchecked, and people tend to accept it as the part of the system. In this paper I would be discussing and deliberating that work place violence particularly verbal abuse is a serious matter and it can have negative consequences not only on person's health but also on the productivity of the employee. This will be followed by some strategies to promote healthy work environment and how can we combat violence at individual, organizational and country level.

\section{Key words}

Verbal violence, Nursing, Workplace violence, Pakistani context

\section{Scenario}

A competent and experienced faculty of middle age group was recently promoted to senior level in an emerging institution. She was loaded with classroom teaching and clinical supervision of multiple classes. She was surprised on this assignment distribution, and raised her concern on this imbalance and biased dissemination of task. During the discussion her concerns were ignored and she was devalued and intimidated. She could not leave this job as her husband died due to some malignant disease and was the only bread earner for her family and kids. The situation further deteriorated when she was periodically criticized for ineffective teaching and threatened for her job security leading to fear, low self-esteem and worthlessness. These negative implications affected her life to such an extent that she was ending up in depression. Based on her traits and excellent teaching style she was respected by all the students and teachers in the institution. She was climbing the professional ladder with a speed that there were high chances of her becoming the future leader of the institution. On the other hand, the head of the institution, was equally talented but had commanding and authoritative leadership style, which was making her indecent and discourteous due to position and power. She resisted change and her short temperedness was making the environment suffocated, tensed, and lacked freedom of expression. 


\section{Introduction}

After having a glimpse of the scenario, it is a growing fact these days that in this modern era all of us are struggling for the theme of going higher, faster and further which is creating competition and resulting in stress and imbalance in our lives. This stress if not handled wisely, is then expressed in the form of violence. Work place violence is the most distressing subject globally, and has been a concern since many decades affecting home and work environment. It affects variety of domains such as hospitals, educational institutions, banks, and multinational companies. This cancer has even invaded health care sector worldwide. Nursing professionals whether they are at hospital or at academics institutions are subjected to violence. Although, it has been a burning issue among nursing health professionals it has often gone unchecked, and people tend to accept it as the part of the system.

In this position paper I would be discussing and deliberating that work place violence particularly verbal abuse is a serious matter and it can have negative consequences not only on person's health but also on the productivity of the employee. This will be followed by some strategies to promote healthy work environment and how can we combat violence at individual, organizational and country level.

\section{Significance of the problem}

It's a logical fact that a healthy and secure work environment is very important where the employees can exercise their rights, have liberty of thoughts and speech, and where their dignity and self-respect is maintained. Gill and Colleagues ${ }^{[1]}$ in their study found that workplace violence is an alarming subject globally, and can have negative implications on organization like decreased employee productivity, self-esteem, and high rate of absenteeism. Workplace violence particularly verbal abuse is a distressing issue, and one of the major contributors of frustration and high turnover rate among nurses. Empirical studies suggest that nurses are subjected up to three times as much violence in the workplace than any other profession ${ }^{[2]}$. Nurses face high levels of verbal abuse from patients, their caretakers, colleagues, bosses, physicians, and the list goes on.

\section{Verbal violence in Pakistani society}

Despite of tons of literature and researches on work place violence, have we ever pondered upon that why verbal abuse is such a critical and serious subject? In Pakistani context, cases of verbal violence are underreported due to fear of retaliation, fear of losing job, lack of confidence, lack of communication and assertive skills, fear of loss of privileges and benefits, and other potential hazardous repercussions. The under reporting factor may be assumed to stem from the behavior where the nurses blame themselves for the abuse instead of the abuser. In addition to it, they also lack the power to prevent such events. This leads nurses to accept verbal violence as the part of the job and the system. Somani and Khowaja ${ }^{[3]}$ segregate work place violence into physical and psychological violence. Psychological violence can be further differentiated into: verbal violence, bullying and sexual violence. Nurses experience both horizontal and vertical violence at workplace. When they encounter violence from other nurses at the same hierarchal level then this kind is called as horizontal violence whereas, if the oppression involves top rigid management hierarchy with most power remaining with nurses at leadership positions and physicians, then this can result in vertical violence.

In this paper, I would be focusing on vertical verbal violence. First, it is important to understand what verbal abuse is? "Verbal abuse is speech that is intended to humiliate or embarrass the target. The recipient feels humiliated, disrespected, and devalued. It includes yelling, making belittling comments, cursing, name calling, and threats” ${ }^{[4]}$ (p. 58). International Council of Nurses defines verbal violence as "behavior that humiliates, degrades or otherwise indicates a lack of respect for the dignity and worth of an individual” ${ }^{55}$ (p. 44). The above mentioned scenario is true reflection of verbal violence where the head of the department humiliated and insulted the staff member when she voiced her concerns on unjustified distribution of workload. Although verbal abuse is expressed through words and tone of voice, however, it also includes 
gestures such as eyes rolling in hatred and pretending not to observe when a colleague is flooded with multiple stressors. In a survey of 950 people researcher found that $82 \%$ of people endorsed dysfunctional behaviors at least weekly ${ }^{[4]}$. The most frequently reported type of behaviors include using threatening or abusive language, humiliating someone in front of others, refusing to mentor, and ignoring attempts at conversations ${ }^{[6]}$. Broome ${ }^{[7]}$ reports a research study done by Robertson in 2004 and Shewchuk in 2005 that verbal violence can also be seen as undue criticism, sarcasm, raised voices and shouting. It has been argued that verbal abuse is less extreme but more wide spread form of work place violence. A study was conducted on 1400 participants representing every region from United States and 17 other countries which included Afghanistan, Taiwan and Saudi Arabia, and it claimed that verbal forms of violence like extreme criticism and hostility, threatening attitude, coercion and harassment are more prevalent than physical ones in health care setup ${ }^{[8]}$. Statistical data from another study reveals that more than $75 \%$ of the participants identified intimidation as the dominant form of work place violence. It is usually under reported as the abuse occurs behind closed doors with no evidences ${ }^{[8]}$. The author further reports that $71 \%$ of the participants were confronting severe criticism from supervisors like physicians, nursing directors and managers ${ }^{[8]}$. It's a bitter truth that although verbal abuse does not leave a physical scar however, the emotional damage done to the inner core and self-worth of the recipient can be very traumatic, which can have potential dangerous outcomes at home and workplace productivity.

\section{Causes of verbal violence}

Work place violence is not an individual problem but it is a complex and structural matter, whose roots are deeply surrounded by organizational, cultural, social and economical factors. Incidences of verbal abuse are under reported. One of the most leading causes of verbal abuse is workplace command and authority. There is a fine line between authoritative behavior and power, and people holding leadership position can switch to power role accidentally or deliberately. Violence and aggression are strongly correlated, and it is researched that vertical violence has been associated with displays of aggression towards someone who has some type of authority over another ${ }^{[6]}$. Sofield and his colleague Salmond ${ }^{[9]}$ talks about other causes of verbal abuse which are highly stressful work situations, and unequal interpersonal relationships and conflicts. Conflicts between the bosses and employees are another most common cause for verbal abuse. Longo ${ }^{[6]}$ describes conflict as "differences about how expected needs will be met, usually manifesting in emotional tension and relational separation or combative behavior". Another crucial reason for verbal violence at work place is incorrect use of power and bad politics which can surface in every human interaction and the actual purpose of its use and manipulation are purposely ignored or misinterpreted ${ }^{[10]}$. Power is also expressed as "individual's ability to influence other in order to achieve goals" ${ }^{[10]}$ (p. 95). The authors brought up a critical point in the statement that power is the basic fuel assisting in transforming the hidden intents into actual picture. Connecting with the scenario the head of the institution's ultimate goal and intention was to retain the leadership position as the key attraction was of financial security, fame, respect, and development of relations with higher leadership personnel's. Hence, she was trying to translate her intentions into reality by demoralizing and belittling the employee to secure her position.

\section{Consequences of verbal abuse}

Verbal violence is common in healthcare organizations in Pakistan. Nursing professionals who experience verbal violence whether employed in hospital setup or academia can face various physical and psychological consequences. The impact of verbal aggression not only threatens employee but also the well-being of other employees working in the organization. Dombrowsky ${ }^{[4]}$ mentions consequences of abuse which include being afraid to speak out, failing to ask questions for fear of ridicule and physical symptoms like headaches, sleep disturbance or pain. Verbal abuse significantly decreases morale, increase job dissatisfaction, and creating hostile work environment ${ }^{[9]}$. Fear reactions are also common that can lead to more serious psychological disorders like anxiety, depression and post-traumatic stress disorder. Other psychological symptoms include inability to communicate with supervisors; colleagues may avoid and neglect the victim. Apart from psychological, behavioral disturbances are also evident such as frustration, anxiety and depression which can result in 
decrease concentration and communication. Sofield and his associate ${ }^{[9]}$ reported that violent behaviors on the part of nursing colleagues led to even more stress for nurses than the violence from physicians or patients. It can lead to absenteeism, staff turnover and resignation if this behavior is persistent and unchanged. If the employee somehow retains in the organization, then long term effects can be evident such as mistrust, low self-esteem, and decrease job satisfaction ${ }^{[9]}$. Associating with the scenario, due to repeated verbal attacks the faculty member was presenting psychological symptoms such as fear of being insulted again on unnecessary things, job insecurity, anxiety, and inability to concentrate on given tasks and her self-esteem and morale was decreased to such an extent that she was ending up in depression. One of the study conducted on nurses in Pakistan shared that disruptive behaviors results in low self-esteem, low confidence, worthlessness, and powerlessness ${ }^{[11]}$. Various studies support that high turnover rate, resignation, and intent to leave profession are linked to verbal abuse ${ }^{[9]}$.

\section{Strategies to combat violence}

The electronic media is crowded with research based literature on different forms of violence, and with advancement there is growing awareness through electronic, print media, television media and social networking. Different literature provides different approaches to deal with violence but the importance lies in the fact that how these strategies are transformed into action as all the steps cannot be implemented due to difference in culture. Strategies have to be designed for both the victim and the perpetrator. For the victim we have to work on the weaknesses. One of the findings from the study suggests that nursing health care professional's lacks skills to respond and cope with verbal abuse ${ }^{[9]}$. In this study the authors have highlighted a critical point that nurses consider and accept verbal abuse as part of the job which can influence in continuation of the problem ${ }^{[9]}$. Indeed, it has become extremely crucial to hit hard on the target i.e. to equip nurses with strategies to deal with verbal violence competently and professionally.

\section{Strategies at individual level}

I will discuss some strategies in light of literature at individual level. At personal level verbal abuse can be responded by following an acronym "ICE TEA" as it guides to counter abuse rationally rather emotionally ${ }^{[4]}$. In this acronym "I" stands for rapid identification, "C" for determining cause of the abuse, "E" control your emotions, " $T$ " means to take control, "E" get everyone on board, and "A" is for after care. Firstly, rapid recognition makes you aware about the abuse so that one can respond intelligently. Secondly, determine the cause of violence. Thirdly, control your emotions as this gives a rational roadmap. Anger has a natural way out it escalates, peaks and then diminishes. Encourage the person to talk about what is bothering. Talking about the problem is better than reacting. Then, take control of the situation. If you can appeal then speak in calm voice and use short sentences. Avoid retaliatory remarks, be patient and keep focused on the matter at hand. Next, get everyone on board and do not try to handle situation all by yourself. Involve some one higher in organization to talk with the perpetrator. Lastly, after care which should not be neglected and the abused person should be allowed to debrief. If de brief is overlooked, it can cause the incident to be repressed leading to emotional damage.

\section{Strategies at organizational level}

Many institutions use education and empowerment strategies to cope with verbal abuse. Educational programs focusing on violence prevention must be made mandatory for all the leaders, employees, and physicians resulting in less chance of violence outcome ${ }^{[8]}$. Assertiveness training program for nurses is a useful method to cope with verbal abuse ${ }^{[12]}$. In addition to it, continuous sessions on effective communication skills should be taken to create healthy environment. Though these skills are taught and learned from the beginning nevertheless, when facing a challenging situation the most elementary approach may be forgotten. Furthermore educational seminars on conflict resolution, stress management and anger management should be conducted frequently ${ }^{[4]}$. A significant viewpoint that all the employees should be educated and introduced to zero tolerance policies and organizational code of conduct at the time of recruitment, orientation classes, 
continuing education classes and monthly institutional update. Another strategy is coaching and counseling of troublesome behaviors which requires change and by having the person commit to the change. Incident reporting and its documentation is another institutional approach to interrupt abusive behavior. The victim hesitates to report the incident due to fear of retaliation and this was evident in the case scenario. Fear of retaliation is one of the major concern and substantial factor for underreporting by employees in developing countries. Those employees who steps ahead and informs incident of disruptive behavior should be supported by the institution thereby disseminating and advocating zero tolerance policy for their employees ${ }^{[6]}$. It is recommended to have a complete written documentation of the event necessary for filing a complaint or legal action ${ }^{[7]}$. Another suggestion in establishing a healthy work environment is through "rounding for outcomes". Daily rounds provide a way both for recognizing employees and also to identify the existence of troublesome behaviors. This design can fit best in hospital setting but for academic environment there should be platforms where individual faculty can ventilate their problems related to working atmosphere. Principle of confidentiality should be maintained strictly. A mentoring system with in the work place environment can build individual strength and enhance skills. A committee can be developed comprising of neutral bodies to discuss critical issues and reach neutral solutions which is agreeable to both the parties.

\section{Recommendation at policy level}

Violent behaviors have gone unchecked for so long that they have often become deep rooted into the culture of an organization. One of the reasons is that the employees are unwilling to report these behaviors ${ }^{[6]}$. After having clear understanding on the concept of verbal violence and its negative consequences on the individual, these behaviors can no longer be ignored. Those institutions who do not take immediate action when verbal abuse occurs causing the nurses to believe that they do not have the power to change it. Hospitals are mandated to provide a healthy work environment, and part of that requirement includes having a plan to address verbal abuse. A strong commitment and accountability lies on the institution to eliminate alarming behaviors, along with cooperation from everyone is imperative. This accountability and cooperation can be accomplished by developing policies to combat such behaviors. The Joint Commission requires having standards defining disruptive behavior and a process for reporting and managing such behaviors ${ }^{[4]}$. Administrators need to demonstrate a concern for the frequency of disruptive behaviors and implement a clearly outlined plan to address them. The organization should formulate a zero-tolerance policy towards these behaviors, and develop the code of conduct which specify those behaviors that are not acceptable ${ }^{[8,13]}$. Prompt action must be taken to interrupt the cycle of verbal abuse. When both victim and perpetrator are nursing professionals but are at different hierarchal level, then there must be clear organizational mandate in the implementation of policies for zero tolerance. In the absence of zero tolerance policies, the organization will not produce desired outcome. The first and crucial step is to take zero tolerance perspective towards implementing a plan of change, and must be followed in all the circumstances. It is crucial to formulate a code of conduct defining all those behaviors that are considered violent. The code should be informed and involve all workers in an organization, including employees, such as nurses, and nonemployees, such as physician ${ }^{[6]}$. Without this enforcement, the code would be meaningless. Therefore, all the members including nursing leaders, chief nursing officers, and hospital administrators, need to be accountable for enforcing and implementing the code.

\section{Policy at country level}

Harassment or violence at workplace is one of the biggest obstacles faced by working women's. Therefore, the Senate and the National Assembly of The Islamic Republic of Pakistan approved and passed 13 point protection against harassment of women at workplace act 2010. This bill aims to create safe working environment free from abuse, harassment and intimidation with a view toward fulfillment of their right with dignity ${ }^{[14]}$. The bill further stresses that within three days of receiving a written complaint the institution inquiry committee should give written receipt of charges or penalties against the perpetrator. 


\section{Conclusion}

It's a high time to reflect that have the nurses left nursing profession in fear of intimidation, harassment, or any type of violence? Will the health- care industry be able to retain nursing professional to overcome staff turnover and professional migration if violence is not controlled? These are serious questions which the entire nursing force needs to work upon. Being a nurse I strongly feel that our leaders must address this issue with vigilance to protect nurses who are our most valued sources. Nursing profession is facing a serious challenge of violence at workplace worldwide. All the nurses have the right to work in a safe and healthy environment and take autonomous decision. Verbal abuses have extreme negative effects on productivity, morale, self-esteem and entire personality. Every nursing professional right from higher leadership position to the lowest must share the responsibility of ensuring safe environment. This problem can be minimized through the promotion of culture which values dignity and respect. Adopting and implementing a zero-tolerance attitude towards these behaviors is one step towards ending these behaviors. However, development of preventive skills is a strong weapon to battle violence at workplace. Safe working environment will automatically attract nurses to have a comeback in the profession.

\section{References}

[1] Gill, M., Fisher, B., \& Bowie, V. Violence at work, causes, patterns and prevention. Oregon, United States of America: Willan Publishing, 2002.

[2] Paterson, B., McCornish, A. G., \& Bradley, P. Violence at work. Nursing Standard. 1999; 13(2): 43-46. PMid:10095589 http://dx.doi.org/10.7748/ns1999.02.13.21.43.c7476

[3] Somani,R.K., \& Khowaja,K. Workplace violence towards nurses: A reality from the Pakistani context. Journal of Nursing Education and Practice. 2012; 2(3): 148-153. http://dx.doi.org/10.5430/jnep.v2n3p148

[4] Dombrowsky,T.A. Responding to verbal Abuse. Nursing. 2012; 58-61. PMid:23076320 http://dx.doi.org/10.1097/01.NURSE.0000421376.75122.4f

[5] Dumont, C., Meisinger, S.,Whitacre, M.J \&Corbin, G. Horizontal violence survey report. Nursing. 2012; 44-49. PMid:22157835 http://dx.doi.org/10.1097/01.NURSE.0000408487.95400.92

[6] Longo, J.Combating Disruptive Behaviors: Strategies to Promote a Healthy Work Environment. The Online Journal of Issues in Nursing. 2010; 15(1).

[7] Broome, B. A., Dealing with sharks and Bullies in the workplace. The ABNF Journal. 2008; 28-30.

[8] Hader, R., ( Workplace violence survey 2008. Nursing Management, 13 - 19. Joint Commission (2008). Sentinel event alert: Behaviors that undermine a culture of safety. Available from: www.jointcommission.org/SentnelEvents/SentinelEventsAlert/sea_40.htm

[9] Sofield, L., \& Salmond,S.W. Workplace violence: A focus on verbal abuse and intent to leave the organization. Orthopedic Nursing. 2003; 22(4): 274-283. http://dx.doi.org/10.1097/00006416-200307000-00008

[10] Sullivan, E. J., \& Decker, P. J. Effective Leadership \& Management in Nursing (5th ed.). New Jersey: Pearson Prentice Hall, 2002.

[11] Lee, M. B., \& Saeed, I. Oppresion and Horizontal Violence: The Case of Nurses in Pakistan. Nursing Forum. 2001; 36(1). http://dx.doi.org/10.1111/j.1744-6198.2001.tb00236.x

[12] Martin, A., Gray, C. \& Adam, A. Nurses Responses to Workplace Verbal Abuse: AScenario Study of the Impact of Situational and Individual Factor. Research and Practice in Human Resource Management. 2007; 15(2): 41-61.

[13] Porto, G., \& Lauve, R. Disruptive Clinician Behavior: A Persistent Threat to Patient Safety. Patient Safety and Quality Healthcare, 2006.

[14] The protection against harassment of women at the workplace act. Acts, Ordinance, president orders and regulations, Government of Pakistan, Islamabad, 2010. 